\title{
Le Trajet du regard ravageur d'Henri Michaux dans Les Ravagés
}

\section{Cheryl Georget Soulodre}

St. Thomas More College, University of Saskatchewan

Et toi qui viens là, qui es-tu, ton oeil comme une tête passée par la fenêtre?
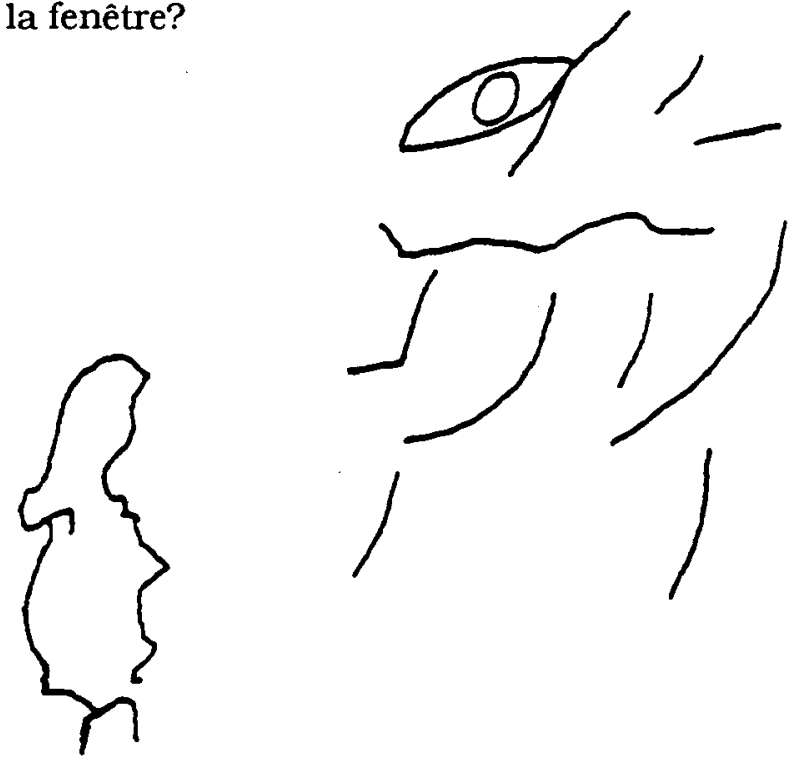

Henrl Michaux, Ebauche: "Ceux qui sont venus à mol," dessin, 1943, légende extraite de Exorcismes, R. Godet, 1943, appartient à M. Robert Godet, Peintures et dessins (Paris: le Point du Jour, 1946), p. 37.

H $\mathrm{n}$ traçant la silhouette d'un monde humanisé-animalisè, qui s'affirme hétéroclite et inversement homogène, Henri Michaux, à l'aide de la parole et du pinceau, envahit l'imaginaire par des méditations sur l'oeuvre picturale "d'aliénés, hommes et femmes en difficulté qui ne purent surmonter l'insurmontable" dans 


\section{LittéRéalité}

Les Ravagés (non paginé). ${ }^{1}$ L'aventure de Michaux, crayonnée dans ce recueil de poèmes en prose, produit une vision fantastique où l'expression écrite engendre et récolte l'oeuvre plastique:

Se montrant, ils se cachent.

Se cachant, ils se montrent (non paginé). ${ }^{2}$

L'acte d'établir le dialogue double de la poésie-peinture, où "L'un regarde L'autre, ils ne se séparent point, ${ }^{3}$ obsède Michaux. Peintre verbal et visuel, il se peint lui-même, afin de nous peindre tous. A cet égard, il nous révèle de son espace coloré: "Peindre, c'est agir, manipuler la pensée." ${ }^{4}$ S'il est vrai que Michaux s'appuie sur la parole et le dessin tout en s'en méfiant, il n'y a pas de doute que son oeuvre livre une activité. Compte tenu de l'épaulement de ces deux domaines, le besoin d'illustrer l'écriture manuscrite de Les Ravagés s'est imposé.

Signalons que Michaux, en disséquant les mots et les coups de pinceau en éléments de plus en plus déguisés, se rapporte à un langage qui "s'achève dans l'inextricable" (p. 61). Poésie haletante, saccadée, sectionnée, Les Ravagés baignent dans un paysage aquatique, un "espace mort" (p. 18), "un espace où plus rien ne bouge" (p. 27), où règne "le calme absolu" (p. 28). Conformément au jeu de l'impasse, l'élément aquatique se présente comme une forme de délire: "Horreur de la possession. Insupportable, indigne possession" (p. 89). On fait de "l'homme ... possédé ... et toujours à reposséder" (p. 69) dont les "forces ont diminué (et qui) vont de plus en plus diminuant," (p. 87) ce qu'on en veut. Dans cette perspective. Michaux nous a donné un avant-goût de la relation entre la fantasmagorie, la tentation et la vérité dans Poteaux d'angle:

Tu laisses quelqu'un nager en toi, aménager en toi, falre du plâtre en toi ... (p. 10).

C'est dire que cet envahissement paralyse la réflexion à la recherche de l'être. D'où son oeuvre Moments qui apporte un autre témoignage:

obstacles ...

Trop de tournants ... 
Interceptions

interceptions (p. 40).

L'énergie de Michaux se redit sans arrêt dans l'emploi de traits spontanés à la limite de l'incohérence. Or, l'accès au monde imaginaire est un monde que Michaux explore, un monde où il vit et revit conflits, un monde où il klivre avant tout et d'emblée, (son) énorme, indicible malaise" (non paginé). Dans l'espace aquatique, il peint la vie angoissée du parcours du regard, témoin intérieur du verbal et du visuel:

La vague, la double, la triple vague, la vague ... porte des yeux ... apporte, emporte, rapporte des yeux, de vastes yeux aux regards de reproche, de ressentiment [...] des yeux qui veulent le mal, des yeux pleins de furie ... (p. 15).

A ce compte, on ne saurait s'étonner du "ravage" que préconise Michaux dans le décor littéraire et pictural. Rage, menaces, violence: le lecteur-observateur le ressent. Certes, "les rapports avec l'entourage seront pénibles" (p. 67). Car le drame de l'existence, Michaux le porte. Et à cela. Michaux nous le transmet. Par le choix délimité des mots fragiles mais chargés de violence dont celle-ci éclate en pétulance verbale, par le visage incognito du poète-dessinateur effacé mais effaré, Michaux ressuscite la composition, la décomposition, la recomposition de l'espace de l'écrit et du dessin.

Modèle de l'oeuvre michaudienne, le poète-peintre fantasme à partir d'un naufrage, "un navire sans occupants, sans équipage, sans agrès, sans rien de cet air allant, commun à tout ce qui navigue» (p. 59). Imitateur et reproducteur du monde réel, - l'obscurcissement de l'esprit, la stérilité de la pensée, les empêchements de la conduite $\longrightarrow$ (p. 60), le naufrage concrétise dans "l'eau: absolument tranquille, ni animée, ni aimée" (p. 17). L'harmonie rompue, "visages enfoncés, engoncés les uns dans les autres ... amas de visages, visages dans le vague ... mangé par un visage est un autre visage" (p. 33), Les Ravagés reflètent «l'humilié, (le) vaincu, ... l'écrasé" (p. 57). Un des textes majeurs sur le Taôisme fait état du principe aquatique: 
Rien dans ce monde n'est plus doux et faible que l'eau; mais pour combattre le difficile et le fort, il n'y a rien comme elle.

Désertant "l'être sans défense" (p. 57), l'eau dans sa force suprême "jamais plus ne se reposera" (p. 13). Peu de choses peuvent la maitriser lorsqu'elle se déforme pour s'écrouler dans "un blanc sans plus, "terra incognita" (p. 66).

L'eau met à l'épreuve l'inexistence réelle de ses "formes animales ou humaines" (p. 61), à l'instar d'un "être sans forme, sans arêtes, sans caractéristiques aucunes, ..... ${ }^{7}$ Expression de l'espace, "Autour, l'eau: ... une eau lourde" (p. 17), Bachelard confirme:

L'appel de l'eau réclame en quelque sorte un don total, un don intime. L'eau veut un habitant. ${ }^{8}$

Or, consentir à la nappe aquatique, n'est-ce pas, pour Michaux, le moyen de s'orienter vers l'échappement? "Et la vie ... ça n'a plus de sens, la vie, tout le contraire, c'est la non-vie qu'il connait, qu'il subit, qu'il voit, le béant de la vie, le gelé de la vie, le mutisme et l'immobilité, l'impénétrabilité des êtres" (p. 66). Cette invitation, bel et bien placée dans le trajet de l'osmose, provoque chez Michaux, une sorte d'hallucination qu'il verbalise.

Dans la peinture écrite de Michaux, la censure se fait jour par "fragments, failles, fissures" (p. 13). Au premier abord, ceux-ci sembleraient avoir été lancés à l'improviste. Mais, le lecteur-observateur bientôt se rend compte que la parole a triché en raison des bribes de mots de vocabulaire qui s'amoncellent dans l'eau humanisée-animalisée. Coupable de trahison par sa puissance, l'étendue d'eau affirme davantage la défiguration de la forme humaine-animale comme un "sol indéfiniment déchiqueté, aux croulantes mottes anonymes, dressées-déjetées" (p. 13). L'eau se retourne contre elle-même: "forme informe" (p. 13), résultat du refus: "le prive de lui" (p. 13), comble du vide: "Incessant écroulement" (p. 13). Créatures "d'une espèce inconnue" (p. 53), les formes animales ou humaines sont réduites à des parcelles, images de "débris" (p. 71). Engagées dans un lieu immense indéterminé, les victimes sont prises par uune mélancolie ..., une détresse de fin de monde, une fatalité irrenversable» (p. 18). Cette demeure-zone coïncide à un visage arrêté, souffle syncopé par le regard traqué de l'homme: 
Chargés d'un dynamisme diabolique et comme boullonnant, les yeux, traversés d'impulsions vampirisantes indiciblement implacables "commandent" (p. 52).

Exercice d'une imagination animalisée-humanisée, la vérité dramatique du monde est le vide, "l'inavouable tout de même avoué" (p. 29).

Poésie exigeante au regard qui gouverne, l'oeuvre de Michaux, est un dialogue prenant entre le poète-peintre et le lecteur-observateur. Zone de la conscience de Michaux où les yeux de partout font visite, l'acte réciproque du regardant et du regardé afflue sa poésie-peinture:

Un guéridon est veillé par deux cygnes. Chaque cygne est veillé par deux ocelots. Chaque ocelot lou panthère ou gros chat tacheté) par deux serpents. Chaque serpent par seize triangles, et se trouvent les triangles sous l'observation d'yeux sans nombre, braqués, scrutateurs (p. 31).

Ceinture de mouvement où court et fourmille un monde extérieur-intérieur, Michaux ne cesse de mettre en scène le personnage du regard obstiné: "il faut tout droit aller à eux les yeux dans les yeux, (et) les forcer de s'exprimer" affirmait-il dans Vents et poussières (p. 73). Echo de l'invasion, l'oeil entreprend son rôle:

les yeux impavides - magistrale discordance, signature

de son mal - font comme si de rien n'était; dans cet

impossible contraire, si contrariant, ils continuent,

ils maintiennent.

L'habitant de la face en désordre n'abandonne pas (p. 20).

L'oeil, libre-champ pour les fantasmes, fait de quelques vers un poème-tableau et de quelques lignes ou taches un tableau-poème:

Ils sont trois dans le tableau.[...]

Même expression: déchargés de toute personnalité, vidés de la particularité d'être une personne. Ainsi 
les peignit l'homme à la vie interceptée: indifférenclés.

La variété du sentir perdue pour lui, perdue pour tous (p. 63).

Significativement, l'oeil, reflet du moi du poète, s'apparente à l'équivalence verbale. Par contre, dans le langage peint de Michaux, il y a contradiction car loeil tend d'une part à séduire et d'autre part à disparaitre des visages esquissés. Dans l'écriture saisissante de Michaux, les formes animales ou humaines se rétractent sous le regard comme des personnages exclus ou écrasés par autrui. L'oeil, champ de conscience du poète, se manifeste par la transmutation d'une multitude de formes mi-humaines, mi-animales, écho de l'occupation de la pensée. Simultanément envahissant-envahisseur, l'oeil clos et ouvert, est un labyrinthe, paysage propice pour les fantasmes et expériences d'animation. Le regard, coulant dans la noirceur, entreprend un voyage dans l'intérieur et s'embarque dans l'exploration de l'inconscient. Hostilité de l'espace, l'oeil le subit:

Dans le visage un oeil qui n'existe plus, comme bu par un buvard. Il en reste le pli. OEll qui a renoncé à être, ne trouvant au dehors rien à sa convenance (p. 73).

Surgissement du tableau-poème, le regard se confie à une synthèse d'évolution. Cet échange interrogateur destiné à peindre le sens premier de Les Ravagés livre un compte-rendu de l'acte violent de l'homme, celui de la métamorphose en ruines durcies du silence dans le "sphinx qui ne répond pas à vos questions, qui sans bouger, nettement, pose 'ses' questions" (p. 12). Le sphinx, grand paradoxe, monstre synonyme de mystère silencieux des âges et de passivité voilée, sert de symtômes de froideur et de dureté vis-à-vis du Je craintif. L'esthétique de l'inconscient chez cette créature fabuleuse est élucidée par le jeu du regard: "le sphinx à l'oeil d'homme garde sa pose qui ne doit plus être dérangée" (p. 12).

Loeil ne connaît pas de repos; Michaux transmet une forme imaginaire à de nombreux instincts agressifs et nuisibles cachés au plus profond de son subconscient. A bien des égards, il se sert du portrait de l'inachèvement comme une forme d'exorcisme à l'instar de Rimbaud:

La violence du venin tord mes membres, me rend difforme, me terrasse. (p. 10) 
Le trouble du dedans chez Michaux s'exprime par les monstres terrifiants qui hantent son univers. Car il peint verbalement le vocable "ravagé»:

Renversé, lézardé, morcelé, toute appartenance humaine oubliée ... (p. 13);

... monstrueux animal-objet, dont l'immobilité ne doit pas tromper ... (p. 79).

Un mode visible de moules ébauchés, images faisant appel au contorsionniste, cette nouveauté violente d'une expressivité particulière renforce la transposition, étiquette de l'homme. Passage d'une forme à une autre, renforcé par la brièveté et la privation des visages, l'homme, attribué à un rôle de travestissement, se conjugue à la neutralité. C'est alors le chevauchement entre l'être et le non-être; il vit comme s'il ne vivait pas. En conséquence, il glisse d'une forme mouvementée à l'anonymat d'une substance. Autrement dit,

Fils et fibres à présent se continuent en

écriture, sur laquelle il revient, la faisant plus

fine, toujours plus fine, la recouvrant, la traversant

de manière qu'elle puisse vraiment échapper à tout

déchiffrement (p. 62).

Ecriture peinte, la technique de Michaux mime la peinture écrite qui offre une ressemblance avec Giacometti; nombreux sont les êtres évanescents. Certains tableaux de Michaux ressemblent plus ou moins à un art figuratif: des croquis de corps amibiens faits vite et de manière floue, des visages en train de se réveiller ou de disparaitre. Ressemblant à des images en rêverie, généralisées et fugitives, ses dessins constituent des champs tentaculaires de petits coups ou mouvements. voire des taches et d'éclaboussures denses et rapides, bref des positions de déséquilibre:

... Formes? Quelles formes? C'est l'informe. son affaire à présent ...

Quant à la couleur $[\ldots]$, c'est le décoloré à présent, son problème....

Le visage ... est tellement pâll qu'il semble avoir èté 
seulement le lieu d'une exhalaison légère, ou

l'emplacement d'un visage perdu.

Pareillement disparaît le corps réduit, dénué

d'èpaisseur, par endroits inachevé ...

Des bras - à quoi bon des bras maintenant? ...

Entre les jambes, un vide remonte sans

s'arrêter jusqu'à la région du coeur, haut entre les

côtes en pleine poitrine qu'il fend par le milieu où

enfin il s'arrête ... (p. 65-66-67).

Esquissé de cette façon, on vit l'individualisme d'une animation curieuse de chacun de ces détails. A l'exemple de son "langage inédit (que René Bertelé a appelé son "espéranto lyrique"), 11 ses dessins exigent un degré de participation consciente dans son monde enfanté comme un voyage intrépide. Le poète comme l'artiste désire se faire tolérer dans son écriture et son art.

Son ensemble de procédés, ancré dans l'inconscient, est passionnant en raison de son intensité:

L'être, un souvenir seulement; approximatif. fragmentaire, difficilement suscité. L'homme (ce qu'il en reste), un rideau, un mince rideau (p. 67).

Même si la facture de l'écriture poétique imite l'écriture picturale, les moyens d'expression de l'artiste, "poète et peintre du fragmentaire, du momentané, ennemi de toute règle, de tout schéma métrique, de tout plan de composition imposé ..." 12 décèlent une nouvelle exégèse du même langage:

Prises et ficelées par les lassos de lignes sans fin, les représentations premières ont disparu totalement (p. 62).

Le long trajet de la langue écrite à partir de 1922 et de la création picturale d'où ses premières taches datent de 1925 et ses premières peintures de 1937 marque le voyage du déterminé.

Les têtes fascinantes de l'homme et de ses visages innombrables sont en lutte aux attaques d'un monde inattendu. Poésie de l'imagination, celle-ci peint la présence souffrante, fiévreuse et désespérante de l'univers. Situé dans le contexte 
pictural, "la main est presque exclusivement au service de l'oeil (regard normal porté sur les objets), de l'oeil intérieur (regard constructeur d'un imaginaire sans cesse à l'affût)." ${ }^{13}$

L'oell qui creuse envahit. L'oeil bénéficie de ses parages pour faire des fantasmes tout à la fois épiant l'adversaire d'un regard particulièrement puissant:

Toujours, chez Michaux alternent ce besoin de voir, de toucher des yeux et d'imaginer. ${ }^{14}$

L'oeil qui touche l'animé-l'inanimé demeure inaltérable, comme on l'a vu à la lecture du sphinx, dont la réputation est celle du silence énigmatique:

'Le Sphinx: ... Mon regard fixe dont rien ne peut dévier, passant à travers les objets, demeure fixé sur un horizon inaccessible ...,'A Gustave Flaubert. 1889. ${ }^{15}$

Chez Michaux, il y a ce besoin constant d'atteindre par les yeux, lesquels représentent pour lui, la communication la plus intérieure de l'être. Dans ses vers esquissés au stylo comme dans ses lignes tracées au pinceau, s'agite un grouillement de l'humanité et de l'animalité, multiplié afin de désigner le regard de chacun. Par l'intermédiaire de l'oeil, thème du dialogue conflictuel peignant l'isolement de l'homme au 20e siècle, l'oeuvre de Michaux capture. L'aventure du poète-peintre dans Les Ravagés, par la multitude de formes quasi-humaines, quasi-animales, univers lavé de formes déchaînées occupant "le paysage froid" (p. 18), "immense et (désert)" (p. 27), se rapproche d'un journal d'expériences vécues par nous tous à l'aube d'hier, d'aujourd'hui et de demain:

Malaise (p. 69); Un malaise profondément situé ...

(11); Embarras. Situation de malaise ajoutée à son

malaise (p. 65); Comment voir sans malaise celui qui vous fait face (p. 76)? 


\section{Notes}

${ }^{1}$ L'édition consultée est la suivante: Les Ravagés (Montpellier: Fata Morgana, 1976). L'oeuvre de Les Ravagés (Montpellier: Fata Morgana, 1976), p. 1-95 a été reproduite dans Chemins cherchés; Chemins perdus; Transgressions (Paris: Gallimard, 1981), p. 7-70.

${ }^{2}$ "Comme il regarde! ... Comme il a peur de regarder! [...]* écrit Michaux (Peintures et dessins, p. 40).

${ }^{3}$ René Micha, "Loin, loin maintenant est l'Un," La Quinzaine littéraire 156 (16-31 janvier 1973), p. 9. Voir l'étude de Renée Riese Hubert, «Paix dans les brisements: Trajectoire verbal et graphique," L'Esprit Créateur XXVI 3 (Fall 1986), p. 72-86.

${ }^{4}$ Max Moreau, "La Poésie, la peinture et le fondement du langage," Revue internationale de philosophie Vingt et unième année (Revue trimestrielle 1967 - Fascicule 1-2, 79-80 Josiah Royce), p. 318.

${ }^{5}$ Voir G. Di San Lazzarro, Klee translated from the Italian by Stuart Hood (New York: Frederick A. Praeger, 1957), p. 18. Klee note dans son Journal: "It is not my task to reproduce appearances for that there is the photographic plate - I want to penetrate into the inmost meaning of the model... my faces are truer than life."

${ }^{6}$ Translated and compiled by Wing-tsit Chan, A Source Book in Chinese Philosophy (Princeton University Press, 1963), p. 174. "The weakest thing in the world overcomes the hardest of all things" (p. 161) déclare Chan.

${ }^{7}$ Georges Poulet, "Henri Michaux," Entre moi et moi: essais critiques sur la conscience de soi (Paris: J. Corti, 1977), p. 232.

${ }^{8}$ Gaston Bachelard, L'Eau et les rêves (Paris: J. Corti, 1942), p. 221. Quelques pages plus loin, Bachelard écrit: Une mer calme est prise d'un soudain courroux. Elle gronde et rugit. Elle reçoit toutes les métaphores de la furie, tous les symboles animaux de la fureur et de la rage. Elle agite sa crinère de lion. Son écume ressemble "à la salive d'un léviathan"; "l'eau est pleine de griffes". Victor Hugo a écrit ainsi, dans Les Travailleurs de la mer, une admirable psychologie de la tempête ... C'est que la psychologie de la colère est, au fond, une des plus riches et des plus nuancées. Elle va de l'hypocrisie et de la lâcheté jusqu'au cynisme et au crime" (p. 230-231). 
${ }^{9}$ Nous relevons la variante de cette citation dans Vents et poussières: "Les serpents sont veillés par seize triangles, et se trouvent les triangles sous lobservation d'yeux sans nombre, braqués et scrutateurs" (p. 38).

${ }^{10}$ Arthur Rimbaud, "Nuit de l'enfer" dans Une saison en enfer, Poésies (Paris: Librairie Générale Française, 1972), p. 176.

${ }^{11}$ Maurice Bruezière, Histoire descriptive de la littérature contemporaine (Paris: Berger-Levrault, 1975), p. 232.

${ }^{12}$ Kurt Leonard, Michaux traduit de lallemand par André Daniel (Paris: P. Tisné, 1967), p. 21.

${ }^{13}$ Jacques Kerno, "Approche d'une facture," Promesse, Henri Michaux numéro spécial XIX-XX (sous le direction de Jean-Louis Houdebine, automne-hiver 1967), p. 105.

${ }^{14}$ Gur Dumur, "Un Aslatique en Barbarie," Le Nouvel Observateur (vendredi 26 octobre 1984), p. 86.

${ }^{15}$ Richard Hobbs, Odilon Redon (Boston: New York Graphic Society, Richard Hobbs, 1977), p. 103. C'est mol qui traduit. 